\title{
Studies on Electronic Charge of the Hydrogen Bond Proton in Model Molecular Systems
}

\author{
Henryk Chojnacki \\ Institute of Physical and Theoretical Chemistry, Laboratory of Quantum Chemistry, I-30, Wrocław \\ University of Technology, Wyb. Wyspiañskiego 27, 50-370 Wrocław, Poland \\ E-mail: chojnacki@kchf.ch.pwr.wroc.pl
}

Received: 31 March 2003 / Accepted: 9 April 2003 / Published: 25 June 2003

\begin{abstract}
The population analysis of the hydrogen bond atoms was analyzed within the different basis sets for model molecular systems for the ground and low-lying excited electronic states. The Mulliken, Löwdin and Hirshfeld methods were used in our investigations. It has been shown that normally the proton is transferred, however, in some excited electronic states the hydrogen atom displacement might be responsible for the tautomeric interconversion.
\end{abstract}

Keywords: hydrogen bonding, proton transfer, population analysis.

\section{Introduction}

The proton transfer reactions are the simplest but very important in many chemical problems as well as in some biological processes. It appears that the low-barrier hydrogen bond (LBHB) for this displacement may play fundamental role in stabilizing intermediates in enzymatic reactions and in energy lowering of transition states [1]. There is considerable evidence that a LBHB may be important in the reaction catalyzed by $\Delta^{5}-3$-ketosteroid isomerase [2]. Recent computational and gas phase experimental studies [3] have shown that LBHB can exist in the gas phase systems as well. The implications of isotope effects have also been studied for structures containing low-barrier hydrogen bonds [4]. On the other hand, the multiple proton transfer seems to play an important role in quantum chemical interpretation at molecular level of some biological processes like heredity, mutations, aging and cancerogenic action [5]. 
It seems that the electron density distribution within the hydrogen bond atoms is of essential importance for considerations of the proton transfer reactions. However, the systematic studies of this property are rather scarce in the present literature. Although theoretical studies are numerous, most of them is concentrated on the ground state properties. On the other hand, mainly Mulliken population analysis is usually performed and no comparison with other methods was done so far.

\section{Model systems}

In our systematic investigations as simple as possible but representative model systems have been chosen. Anion $\mathrm{H}_{3} \mathrm{O}_{2}{ }^{-}$, water dimer $\mathrm{H}_{4} \mathrm{O}_{2}$, and $\mathrm{H}_{5} \mathrm{O}_{2}{ }^{+}$cation were studied. Furthermore, malonaldehyde $\mathrm{C}_{3} \mathrm{H}_{4} \mathrm{O}_{2}$ with intramolecular hydrogen bonding and imidazole dimer have been investigated. In all cases the full geometry optimization in the ground and excited states was performed.

In some earlier papers the above mentioned ions were studied at the multireference level $[6,7]$, whereas malonaldehyde at the DFT level [8]. Imidazole chain has also been considered in relation with its electrical properties [9].

\section{Calculational Outline}

The non-dynamical correlation energies were evaluated for the two extremal proton positions of the model systems within the hydrogen bond. The population analysis was performed within the Mulliken, Löwdin and Hirshfeld formalisms. The calculations of the atomic charges for the excited electronic states were performed within the CIS approach. For the above-mentioned model hydrogen bonded systems all CAS SCF [10] calculations have also been performed with frozen 1s and $2 \mathrm{~s}$ orbitals

The Hirshfeld idea to describe a molecule is to divide it into atoms and examine how these differ from the free atoms [11] i.e. this formalism is based on the total electron density $\rho$ and atomic densities $\rho_{\mathrm{i}}$ of the i-th atom. On the contrary to the Mulliken population analysis, it can be given the physical meaning. Here, the atomic charge of the $\alpha$-atom is expressed as

$$
\mathrm{q}_{\alpha}=\int \rho_{\mathrm{i}}(\mathrm{r}) \mathrm{W}_{\alpha}(\mathrm{r}) \mathrm{dr}
$$

where

$$
\rho_{\mathrm{i}}(\mathrm{r})=\rho_{\mathrm{o}}(\mathrm{r})-\Sigma \rho_{\alpha}\left(\mathrm{r}-\mathrm{R}_{\alpha}\right)
$$

denotes the relevant deformation density and $\rho_{\alpha}\left(r-R_{\alpha}\right)$ is the electron density of isolated atom situated at the $\mathrm{R}_{\alpha}$. Furthermore,

$$
\mathrm{W}_{\alpha}(\mathrm{r})=\rho_{\alpha}\left(\mathrm{r}-\mathrm{R}_{\alpha}\right) / \Sigma \rho_{\beta}\left(\mathrm{r}-\mathrm{R}_{\beta}\right)
$$

is the respective statistical weight. Those relationships can be derived from the well-grounded information-theoretical formalism $[12,13]$.

The numerical calculations were performed by using mainly GAUSSIAN98 [14] as well as GAMESS-US (VERSION 6), and CERIUS2_4.5 computer programs. 


\section{Results and Concluding Remarks}

An essential role of basis set has been documented in our considerations of population analysis for hydrogen bonded systems. It was illustrated in the case of charge on the hydrogen bond proton of the malonaldehyde molecule (Table 1), where in the case of Mulliken population, dramatic differences were found. It should be noted that the results are strongly dependent on the molecular geometry where its optimization is very important (Table 2) as well.

In general, much less charge values are obtained according to Löwdin and Hirshfeld formalism in particular (Tables 3 and 4) than those resulting from the Mulliken population. In the latest case they remind those obtained by using multiconfigurational wave function [10]. On the other hand, the Hirshfeld method, based on information theory, seems to be the best grounded population analysis. Generally, in the ground state the electron charge on the proton is enhanced for its middle position within the hydrogen bond in comparison with the endpoint positions. It seems that the smooth changes on the ground potential curve are followed by the tempered changes of the proton charge [7]. However, the curves representing the charge distribution are more sensitive to wave function variations than the corresponding potential energy curves.

Table 1. Mulliken ground state hydrogen bond proton charges in malonaldehyde within the different basis sets. In parentheses the respective values for the middle hydrogen bond proton position are given

\begin{tabular}{lc}
\hline Basis Set & Charge \\
\hline 6-31G & $0.486(0.402)$ \\
DUIJNEVELDT $^{\text {a) }}$ & $0.637(0.666)$ \\
SADLEJ $^{\text {b) }}$ & $0.159(0.272)$ \\
HC $^{\text {c) }}$ & $0.001(0.406)$ \\
CC-PVQZ $^{*}$ & $0.316(0.361)$
\end{tabular}

a) F.B. van Duijneveldt, Gaussian basis set for the atoms $\mathrm{H}-\mathrm{Ne}$ for use in molecular calculations, IBM J. Res. Dev., (1971) 945.

b) Sadlej basis set [14]

c) Modified Duijneveldt basis set.

Table 2. Mulliken proton charges for standard equilibrium (EQ) and middle (TR) hydrogen bond proton positions of malonaldehyde within the Sadlej [14] basis set in the case of the lowest singlet and triplet electronic states

\begin{tabular}{|c|c|c|c|c|c|c|c|c|}
\hline \multirow[b]{2}{*}{ State } & \multicolumn{4}{|c|}{ Singlets } & \multicolumn{4}{|c|}{ Triplets } \\
\hline & $\mathrm{EQ}^{*}$ & $\mathrm{TR}^{*}$ & EQ & $\mathrm{TR}$ & $\mathrm{EQ}^{*}$ & $\mathrm{TR}^{*}$ & EQ & TR \\
\hline 1 & -0.0907 & -0.3756 & -0.1774 & 0.1088 & 0.1015 & 0.4194 & -0.0765 & -0.0763 \\
\hline 2 & 0.1122 & 0.4071 & 0.1076 & -0.1771 & 0.0917 & 0.4127 & -0.1934 & -0.1941 \\
\hline 3 & 0.0661 & 0.3532 & -0.6700 & -0.0708 & & & & \\
\hline
\end{tabular}

* Calculation performed without geometry optimization in the relevant excited state. 
Table 3. Atomic charges for the imidazole molecule according to Mulliken, Löwdin and Hirshfeld methods (in parentheses the respective values for the middle hydrogen bond proton position are given)

\begin{tabular}{cccc}
\hline Atom & Mullken & Löewdin & Hirshfeld \\
\hline N1 & $-0.471(-0.450)$ & $-0.382(-0.396)$ & $-0.051(-0.100)$ \\
C2 & $-0.032(0.016)$ & $0.034(0.010)$ & $0.032(0.025)$ \\
N3 & $-0.461(-0.465)$ & $-0.315(-0.319)$ & $-0.151(-0.102)$ \\
C4 & $-0.187(-0.183)$ & $-0.111(-0.117)$ & $-0.041(-0.039)$ \\
C5 & $0178(-0.151)$ & $-0.112(-0.131)$ & $-0.027(-0.034)$ \\
H6 & $0.502(0.406)$ & $0.321(0.393)$ & $0.098(0.112)$ \\
H7 & $0.294(0.291)$ & $0.193(0.190)$ & $0.044(0.043)$ \\
H8 & $0.253(0.259)$ & $0.182(0.182)$ & $0.043(0.044)$ \\
H9 & $0.281(0.277)$ & $0.191(0.188)$ & $0.052(0.050)$ \\
\hline
\end{tabular}

Table 4. Mulliken and Hirshfeld ground state HB proton charges in model systems within 6-31G** basis set for standard (EQ) and middle hydrogen bond proton positions (TR)

\begin{tabular}{cccccc}
\hline System & $\mathrm{R}_{\mathrm{e}}(\AA)$ & EQ & TR & EQ & TR \\
\hline$\left(\mathrm{H}_{4} \mathrm{O}_{2}\right)_{2}$ & 2.925 & 0.445 & 0.371 & 0.097 & 0.106 \\
$\left(\mathrm{H}_{3} \mathrm{O}_{2}^{-}\right)_{2}$ & 2.740 & 0.375 & 0.402 & 0.028 & 0.032 \\
$\left(\mathrm{H}_{5} \mathrm{O}_{2}^{+}\right)_{2}$ & 2.416 & 0.494 & 0.462 & 0.161 & 0.166 \\
$\left(\mathrm{C}_{3} \mathrm{H}_{4} \mathrm{O}_{2}\right)$ & 2.553 & 0.479 & 0.462 & 0.123 & 0.124 \\
$(\text { Imidazole })_{2}$ & 2.861 & 0.395 & 0.379 & 0.170 & 0.214 \\
\hline
\end{tabular}

The rearrangement of the electron density distribution in excited electronic states influences greatly the proton position and its electron charge (Table 5). It seems that at least in some cases, in excited states the hydrogen atom transfer within the hydrogen bond might be responsible for the tautomeric interconversion reactions.

Table 5. Mulliken and Lőwdin hydrogen bond proton charges in model systems within the 6-31G** basis set for ground and lowest singlet excited state. In parentheses the respective values for the middle hydrogen bond proton position are given

\begin{tabular}{ccc}
\hline System & Mulliken & Löwdin \\
\hline & Ground State & \\
$\mathrm{H}_{3} \mathrm{O}_{2}^{-}$ & $0.195(0.572)$ & $0.045(0.139)$ \\
$\mathrm{C}_{3} \mathrm{H}_{4} \mathrm{O}_{2}$ & $0.494(0.588)$ & $0.315(0.335)$ \\
$(\text { Imidazole })_{2}$ & $0.751(0.748)$ & $0.348(0.348)$ \\
& Lowest Singlet Excited State & \\
$\mathrm{H}_{3} \mathrm{O}_{2}^{-}$ & $0.401(0.507)$ & $0.067(0.126)$ \\
$\mathrm{C}_{3} \mathrm{H}_{4} \mathrm{O}_{2}$ & $0.477(0.565)$ & $0.305(0.323)$ \\
$(\text { Imidazole })_{2}$ & $0.759(0.768)$ & $0.342(0.342)$ \\
\hline
\end{tabular}




\section{Acknowledgement}

This work has been sponsored in part by the Polish State Committee for Scientific Research. The numerical calculations were performed in part in Wrocław Networking and Supercomputing Center by using GAMESS-US (VERSION 6), GAUSSIAN98 and CERIUS2_4.5 computer programs.

\section{References}

1. Cleland, D.; Kreevoy, M.W. Science 1994, 264, 1887.

2. Warshel, A.; Papazyan, A. Science 1995, 269, 102.

3. Garcia-Viloca, M.; Gonzales-Lafont, M.; Lluch, J.M. J. Am. Chem. Soc. 1997, 119, 1081.

4. Huskey, W.P. J. Am. Chem. Soc. 1996, 118, 1663.

5. Löwdin, P.O. Adv. Quantum Chem. 1965, 2, 213.

6. Roszak, S.; Kaldor, U.; Chapman, D.A.; Kaufman, J.J. J. Phys. Chem. 1992, 96, 2123.

7. Kowal, M.; Roszak, S.; Leszczyński, J. J. Chem. Phys. 2001, 114, 8251.

8. Barone, V.; Adamo, C. J. Chem. Phys. 1996, 105, 11007.

9. Brédas, J.L.; Poskin, M.P.; Delhalle, J.; André, J.M.; Chojnacki, H. J. Phys. Chem. 1982, $88,5882$.

10. Chojnacki, H. unpublished results.

11. Hirshfeld, F.L. Theor. Chim. Acta 1977, 44, 129.

12. Nalewajski, R.F.; Parr, R.G. J. Phys. Chem. A 2001, 105, 7391.

13. Nalewajski, R.F. Phys. Chem. Chem. Phys. 2002, 4, 1.

14. Frisch, M.J.; Trucks, G.W.; Schlegel, H.B.; Scuseria, G.E.; Robb, M.A.; Cheeseman, J.R.; Zakrzewski, V.G.; Montgomery, J.A.; Jr., Stratmann, J.R.; Burant, J.C.; Dapprich, S.; Millam, J.M.; Daniels, A.D.; Kudin, K.N.; Strain, M.C.; Farkas, O.; Tomasi, J.; Barone, V.; Cossi, M.; Cammi, R.; Mennucci, B.; Pomelli, C.; Adamo, C.; Clifford, S.; Ochterski, J.; Petersson, G.A.; Ayala, P.Y.; Cui, Q.; Morokuma, K.; Malick, D.K.; Rabuck, A.D.; Raghavachari, K.; Foresman, J.B.; Cioslowski, J.; Ortiz, J.V.; Baboul, A.G.; Stefanov, B.B.; Liu, G.; Liashenko, A.; Piskorz, P.; Komaromi, I.; Gompers, R.; Martin, R.L.; Fox, D.J.; Keith, T.; Al-Laham, M.A.; Peng, C.Y.; Nanayakkara, A.; Challacombe, M.; Gill, P.M.W.; Johnson, B.; Chen, W.; Wong, M.W.; Andres, J.L.; Gonzales, C.; Head-Gordon, M.; Replogle, E.S.; Pople, J.A. Gaussian 98, Revision A.9; Gaussian: Pittsburgh, PA, 1998.

15. Sadlej, A.J. Theor. Chim. Acta 1991, 79, 123.

16. Chojnacki, H. Bull. Pol. Acad. Sci. Chem. 2002, 50, 501.

(C) 2003 by MDPI (http://www.mdpi.org). Reproduction for noncommercial purposes permitted. 